\title{
Slow Particle Production in Nucleus-Nucleus Collisions at Relativistic Energies
}

\author{
Mir Hashim Rasool1*, Mohammad Ayaz Ahmad², Shafiq Ahmad1 \\ ${ }^{1}$ Department of Physics, Aligarh Muslim University, Aligarh, India \\ ${ }^{2}$ Physics Department, Faculty of Science, University of Tabuk, Tabuk, Saudi Arabia

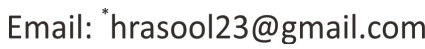

Received 9 November 2015; accepted 8 January 2016; published 13 January 2016

Copyright (C) 2016 by authors and Scientific Research Publishing Inc.

This work is licensed under the Creative Commons Attribution International License (CC BY).

http://creativecommons.org/licenses/by/4.0/

(c) () Op Open Access

\begin{abstract}
In this paper an effort has been made to study the general characteristics of slow particles produced in the interactions of ${ }^{32} \mathrm{~S}$-Em at $200 \mathrm{AGeV}$ to extract the information about the mechanism of particle production. The results have been compared with the experimental results obtained by other workers. The multiplicity distributions of the slow target associated particles (black, grey and heavy tracks) produced by ${ }^{32} \mathrm{~S}$-beam with different targets have been studied. Also several types of correlations among them have been investigated. The variation of the produced particles with projectile mass number and target size has been studied. Also the multiplicity distributions of slow particles with NBD fits are presented and scaling multiplicity distributions of slow particles produced have been studied in order to check the validity of KNO-scaling.
\end{abstract}

\section{Keywords}

Relativistic Heavy-Ion Collisions, Nuclear Emulsion, Multiplicity Distribution, Multiplicity Correlations, KNO Scaling, Negative Binomial Distribution

\section{Introduction}

The study of relativistic nucleus-nucleus (A-A) collisions has attained peculiar importance during the last few decades. In nucleus-nucleus collisions it is important to achieve complete information regarding the mechanism of particle production. When an energetic projectile collides with targets of nuclear emulsion, a number of charged and uncharged particles are produced. The emergence of these particles occurs in a very short time and after this the nucleus remains excited for quite a long time on nuclear scale. The nucleus then de-excites resulting in the emission of a large number of nucleons and other heavy fragments. Usually, the particles emitted

\footnotetext{
*Corresponding author.
} 
through this process of evaporation appear as black tracks as well as low energy grey tracks in nuclear emulsion. So far, very little work has been carried out in the target fragmentation region. For the study of this region it is essential to have experimental data on low and medium energy particles produced due to the processes of rescattering and cascading. In this direction, the EMU01 and other experiments have made considerable effort to understand the role of slow target related particles in nucleus-nucleus collisions [1] [2].

The multiplicity of charged particles in high energy nucleus-nucleus interactions is an important parameter which indicates how many particles are produced in that interaction. The multiplicity distributions of produced particles or emitted particles help in learning the interaction mechanism. Generally, it is accepted that in high energy nucleus-nucleus collisions, the emission of slow target-associated particles (i.e. black tracks) and other heavier fragments takes place at a still latter stage with range $L \leq 3 \mathrm{~mm}$, relative velocity $\beta<0.3$ and energies less than $30 \mathrm{MeV}$. The emission of fast target associated particles mostly the knocked out protons known as grey particles, takes place at a relatively latter stage of the collision. These fast protons with range $L \geq 3 \mathrm{~mm}$ and relative velocity $0.3 \leq \beta \leq 0.7$ lie in the energy range 30 to $400 \mathrm{MeV}$. Moreover, these target-associated particles are mostly slow and fast protons and grey particles are often assumed to be the measure of the number of encounters made by the incident hadron inside the target nucleus [3] and believed to be produced as a result of process of rescattering in the target spectator region. The black and grey tracks taken together are known as heavily ionizing tracks denoted by $N_{h}$.

Our objective in this paper is to employ the interactions of ${ }^{32} \mathrm{~S}$ in nuclear emulsion. The emulsion has the unique property of acting simultaneously as the target as well as the detector, for registering all the charged particles in $4 \pi$ geometry with the highest spatial resolution as compared to the electronic detectors. We have reported some results based on the general characteristics of slow particles produced in the interactions of ${ }^{32} \mathrm{~S}-\mathrm{Em}$ $200 \mathrm{AGeV} / \mathrm{c}$ to extract the information about the mechanism of particle production. The multiplicity distributions of the slow target associated particles (black, grey and heavy tracks) produced by ${ }^{32} \mathrm{~S}$-beam with different targets have been studied. Also several types of correlations among them have been investigated. The variation of the produced particles with projectile mass number and target size has been studied. Some results have also been obtained on the angular distribution of black and grey tracks and values of F/B ratio for these distributions have also been presented. Also the multiplicity distributions of slow particles with NBD fits are presented and scaling multiplicity distributions of slow particles produced have been studied in order to check the validity of KNO-scaling.

\section{Experimental Techniques}

In the present experiment we have used two stacks of Ilford G5 nuclear emulsion plates exposed horizontally to a ${ }^{32}$ S-beam at $200 \mathrm{AGeV}$ from Supper Proton Synchrotron, SPS at CERN for data collection. The scanning of the plates is performed with the help of Leica DM2500M microscope with a 10X objective and 10× ocular lens provided with semi-automatic scanning stages. The method of line scanning was used to collect the inelastic ${ }^{32} \mathrm{~S}$-Em interactions. The interactions collected from line scanning were scrutinized under an optical microscope (Semi-Automatic Computerized, Leica DM6000M) with a total magnification of $10 * 100$ using 10× eyepiece and $100 \times$ oil immersion objective. The measuring system associated with it has $1 \mu \mathrm{m}$ resolution along $\mathrm{X}$ and $\mathrm{Y}$ axes and $0.5 \mu \mathrm{m}$ resolution along the Z-axis. The detailed discussion about the present experiment can be found in our earlier publications [4]-[7].

\section{Results and Discussion}

\subsection{Multiplicity Distributions}

The analysis of the experimental data in terms of multiplicity distributions for different emitted secondaries (slow and fast protons) is one of the main sources of information about the mechanism of particle production. Figures 1(a)-(c) shows the multiplicity distributions of black, grey and heavily ionizing particles from ${ }^{32} \mathrm{~S}$ Emulsion interactions at $200 \mathrm{AGeV}$ along with the distribution obtained from ${ }^{28} \mathrm{Si}-\mathrm{Em}$ [8] and ${ }^{16} \mathrm{O}-\mathrm{Em}$ [9] interactions at $14.6 \mathrm{AGeV}$ and $200 \mathrm{AGeV}$ respectively for comparison. It is observed from the figures that the peaks of the distributions appear in the lower values of $N_{b}, N_{g}$, and $N_{h}$. These distributions seem to be independent of incident energy as well as projectile mass within statistical errors up to lower values of $N_{b}, N_{g}, N_{h}$. This result is consistent with those obtained by other workers [8]-[13]. It may also be noticed from the figures that the per- 


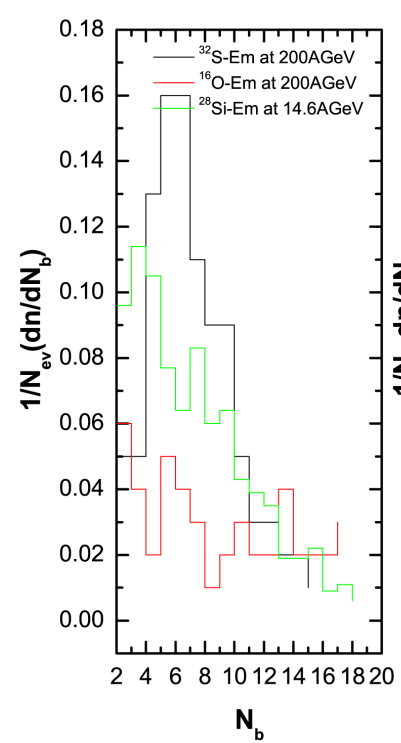

(a)

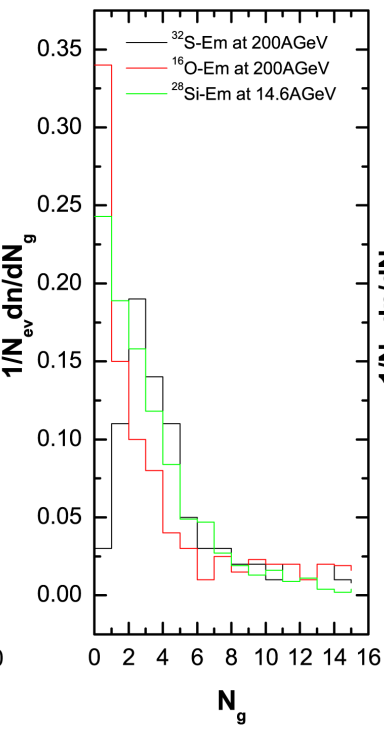

(b)

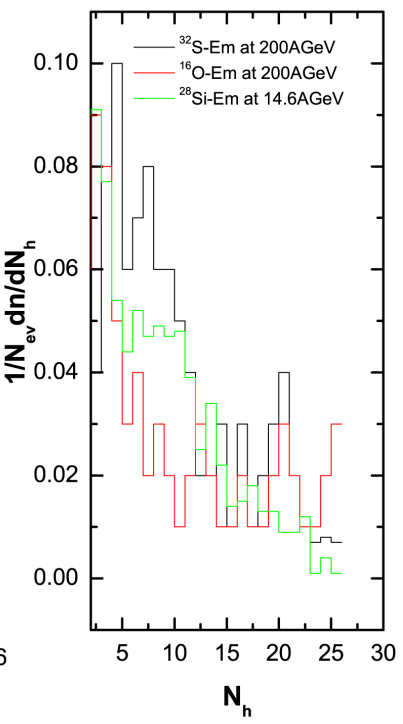

(c)

Figure 1. Multiplicity distributions of secondary charged particles produced in interactions of projectiles with emulsion for (a) black particles (b) grey particles and (c) heavily ionizing particles.

centage of events with large values of $N_{b}, N_{g}$, or $N_{h}$ increases with projectile mass. Finally, it may be concluded from the multiplicity distributions of slow and fast protons produced in nucleus-nucleus interactions that no significant differences are observed regarding the mechanism of their production with energy.

Figures 2(a)-(c) and Figures 3(a)-(c) show the $N_{b}, N_{g}$, and $N_{h}$ multiplicity distributions from ${ }^{32} \mathrm{~S}-\mathrm{AgBr}$ and ${ }^{32} \mathrm{~S}$-CNO interactions at $200 \mathrm{AGeV}$. The results obtained by other workers for ${ }^{12} \mathrm{C}$ and ${ }^{28} \mathrm{Si}$ at 4.5 and 14.6 $\mathrm{AGeV}[8]$ respectively are incorporated in the same figure for comparison. From the figures, it may be observed that the characteristics feature of the distribution is similar in shape for all targets, but the multiplicity range or decay tail increases with target size. It has also been found that the distributions for ${ }^{32} \mathrm{~S}-\mathrm{AgBr}$ are broader than those for ${ }^{32} \mathrm{~S}-\mathrm{CNO}$ interactions. This may reflect the effect of the target mass number on the number of collisions of ${ }^{32} \mathrm{~S}$ beam with target nuclei. Experimental results obtained by other workers [8] [10] reproduce qualitatively similar results.

\subsection{Scaling of Grey Particles}

The possibility of scaling, i.e., similarity in the multiplicity distributions of grey tracks produced in hadron-nucleus and nucleus-nucleus interactions has also been examined. In the present analysis, the events with $N_{g}=0$ have been excluded because the coherent processes may also contribute to such events. Figure 4(a) shows the $N_{g} /\left\langle N_{g}\right\rangle$-distribution from ${ }^{32} \mathrm{~S}$-Em interactions at $200 \mathrm{AGeV}$. A straight line of the form:

$$
\ln \left(N_{e v}\right)=A\left(N_{g} /\left\langle N_{g}\right\rangle\right)+B
$$

is found to represent the present data, $N_{e v}$ denotes the number of events in the given bin and $A$ and $B$ are constants. The best fit to the data is given as:

$$
\ln \left(N_{e v}\right)=-(0.97 \pm 0.07) N_{g} /\left\langle N_{g}\right\rangle+(4.62 \pm 0.09)
$$

For comparison the $N_{g} /\left\langle N_{g}\right\rangle$-distributions from ${ }^{28} \mathrm{Si}$-Em at $14.6 \mathrm{AGeV}$ and $4.5 \mathrm{AGeV}$ and ${ }^{12} \mathrm{C}-\mathrm{Em}$ at 4.5 $\mathrm{AGeV}$ [14] are also plotted in Figures 4(b)-(d). The values of the slopes for ${ }^{28} \mathrm{Si}-\mathrm{Em}$ at $14.6 \mathrm{AGeV}$ and ${ }^{28} \mathrm{Si}$-Em and ${ }^{12} \mathrm{C}$-Em interactions at $4.5 \mathrm{AGeV}$ are found to be $-0.88 \pm 0.04,-0.89 \pm 0.08$ and $-0.86 \pm 0.06$ respectively. The slope parameters are $\sim 0.90$, which are very much consistent with the value obtained for ${ }^{32} \mathrm{~S}$-Em at 200 AGeV. The constancy in the values of slopes for nucleus-nucleus collisions at different energies may be interpreted as existence of some kind of scaling for the production of grey tracks. 


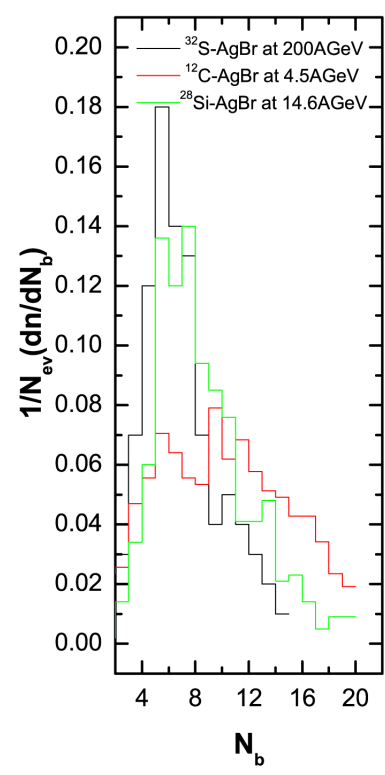

(a)

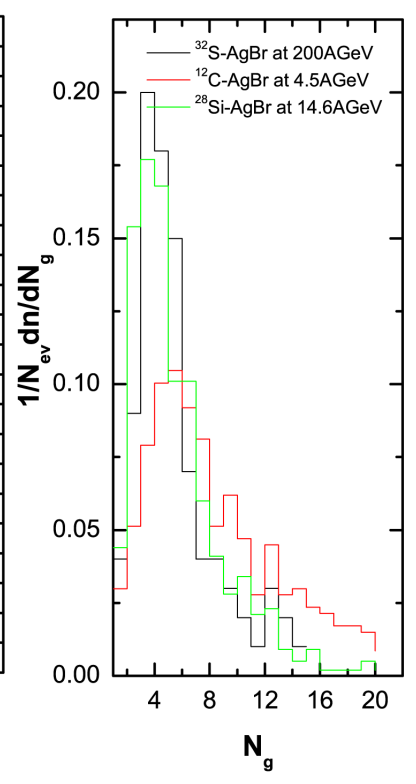

(b)

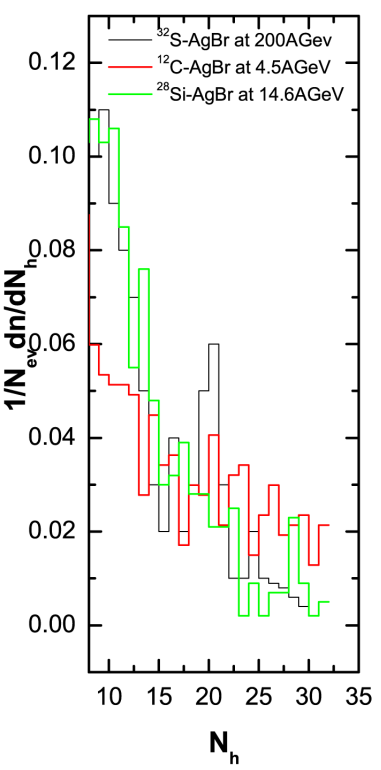

(c)

Figure 2. Multiplicity distributions of secondary charged particles produced in interactions of different projectiles with $\mathrm{AgBr}$ for (a) black particles (b) grey particles and (c) heavily ionizing particles.

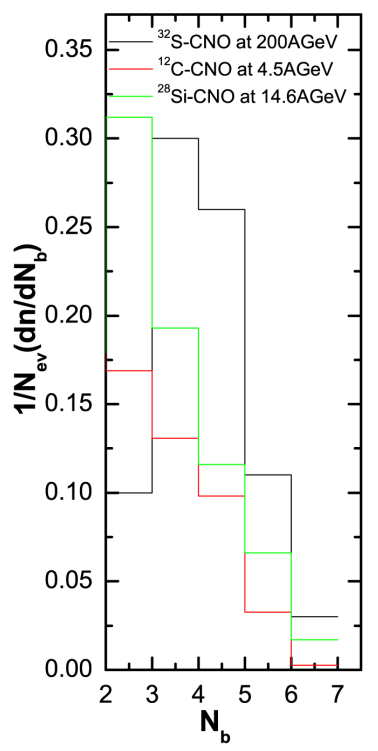

(a)

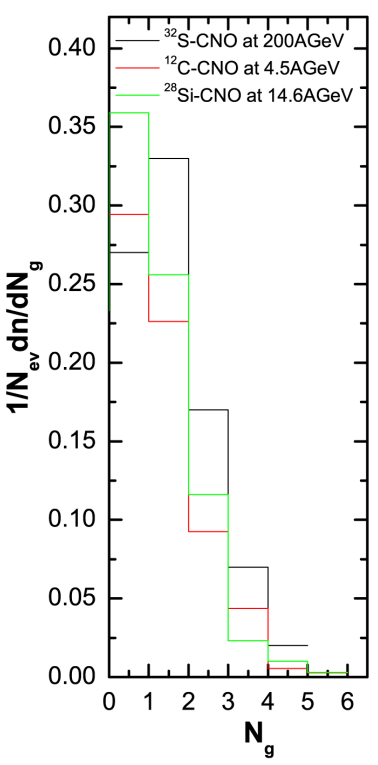

(b)

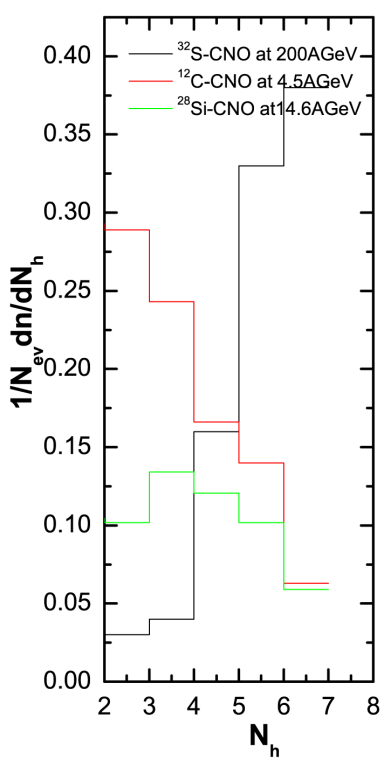

(c)

Figure 3. Multiplicity distributions of secondary charged particles produced in interactions of different projectiles with CNO for (a) black particles (b) grey particles and (c) heavily ionizing particles.

\subsection{Mean Multiplicity of Secondary Particles}

The mean multiplicity is the average number of charged particles produced in various types of high-energy heavy ion collisions. Multiplicity of different charged particles is helpful in understanding the mechanism of multiparticle production. The average values of number of black, grey and heavily ionizing particles produced in ${ }^{32} \mathrm{~S}$-Em interactions at $200 \mathrm{AGeV}$ are displayed in Table 1. The values of $\left\langle N_{b}\right\rangle,\left\langle N_{g}\right\rangle$ and $\left\langle N_{h}\right\rangle$ measured in ${ }^{32} \mathrm{~S}$-Em interactions at $200 \mathrm{AGeV}$ by S. Dhamija et al. [2] and A. Dabrowska et al. [15] along with other results at different energies [8]-[10] [12] [13] [16]-[18] are also listed in Table 1 for the sake of comparison. It is 


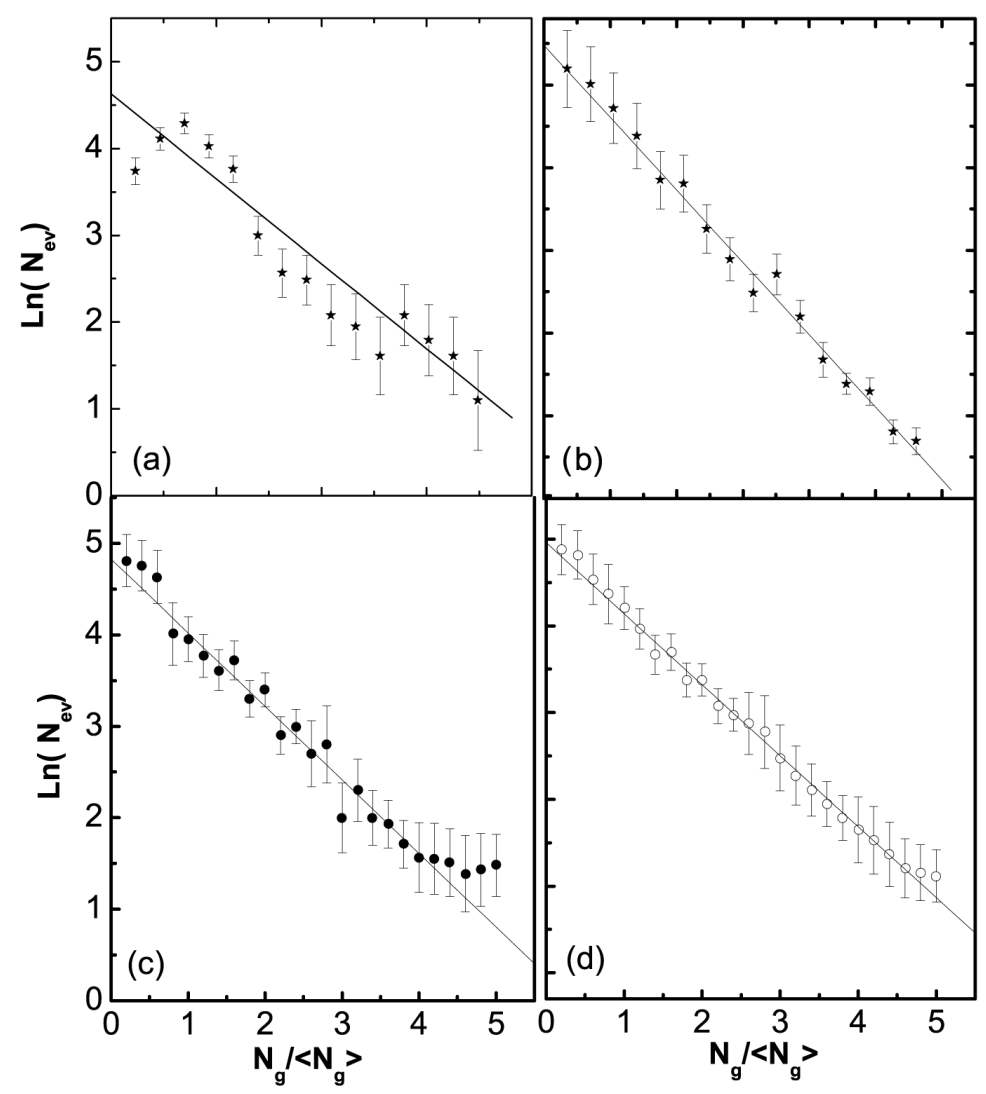

Figure 4. Plot of $\ln \left(N_{e v}\right)$ vs $N_{g} /\left\langle N_{g}\right\rangle$ for different interactions at different energies. (a) ${ }^{32} \mathrm{~S}$-Em at $200 \mathrm{AGeV}$; (b) ${ }^{32} \mathrm{Si}-\mathrm{Em}$ at $14.6 \mathrm{AGeV}$; (c) ${ }^{32} \mathrm{Si}-\mathrm{Em}$ at $4.5 \mathrm{AGeV} ;(\mathrm{d}){ }^{32} \mathrm{C}-\mathrm{Em}$ at $200 \mathrm{AGeV}$.

Table 1. Mean multiplicities of various particles produced in heavy ion collisions at high energies.

\begin{tabular}{|c|c|c|c|c|c|}
\hline Energy/nucleon (A GeV) & Collision Type & $\left\langle N_{b}\right\rangle$ & $\left\langle N_{g}\right\rangle$ & $\left\langle N_{h}\right\rangle$ & Ref. \\
\hline 4.5 & $\mathrm{p}-\mathrm{Em}$ & $3.77 \pm 0.08$ & $2.81 \pm 0.06$ & $6.51 \pm 0.10$ & [18] \\
\hline 4.5 & $\alpha$-Em & $4.70 \pm 0.20$ & $4.70 \pm 0.20$ & $9.40 \pm 0.23$ & [10] \\
\hline 4.5 & ${ }^{6} \mathrm{Li}-\mathrm{Em}$ & $4.91 \pm 0.30$ & $4.23 \pm 0.21$ & $9.14 \pm 0.37$ & {$[17]$} \\
\hline 4.5 & ${ }^{7} \mathrm{Li}-\mathrm{Em}$ & $5.37 \pm 0.18$ & $3.80 \pm 0.12$ & $9.14 \pm 0.29$ & [17] \\
\hline 4.5 & ${ }^{12} \mathrm{C}-\mathrm{Em}$ & $4.51 \pm 0.10$ & $5.75 \pm 0.11$ & $10.26 \pm 0.15$ & {$[8]$} \\
\hline 3.7 & ${ }^{32} \mathrm{~S}-\mathrm{Em}$ & $6.92 \pm 0.25$ & $3.54 \pm 0.13$ & $10.46 \pm 0.37$ & [12] \\
\hline 2.1 & ${ }^{14} \mathrm{~N}-\mathrm{Em}$ & $4.57 \pm 0.21$ & $5.29 \pm 0.31$ & $9.86 \pm 0.25$ & [13] \\
\hline 4.5 & ${ }^{28} \mathrm{Si}-\mathrm{Em}$ & $5.26 \pm 0.09$ & $6.33 \pm 0.10$ & $11.59 \pm 0.14$ & [8] \\
\hline 14.6 & ${ }^{28} \mathrm{Si}-\mathrm{Em}$ & $5.55 \pm 0.08$ & $2.85 \pm 0.06$ & $8.40 \pm 0.09$ & {$[8]$} \\
\hline 60 & ${ }^{16} \mathrm{O}-\mathrm{Em}$ & $4.91 \pm 0.34$ & $2.16 \pm 0.15$ & $7.07 \pm 0.40$ & [9] \\
\hline 200 & ${ }^{16} \mathrm{O}-\mathrm{Em}$ & $5.39 \pm 0.29$ & $2.03 \pm 0.11$ & $7.42 \pm 0.33$ & [9] \\
\hline 200 & ${ }^{32} \mathrm{~S}-\mathrm{Em}$ & $5.04 \pm 0.15$ & $3.67 \pm 0.11$ & $8.71 \pm 0.18$ & [2] \\
\hline 200 & ${ }^{32} \mathrm{~S}-\mathrm{Em}$ & $5.10 \pm 0.20$ & $3.60 \pm 0.20$ & $8.70 \pm 0.28$ & [15] \\
\hline 200 & ${ }^{32} \mathrm{~S}-\mathrm{Em}$ & $6.67 \pm 0.14$ & $3.14 \pm 0.10$ & $9.81 \pm 0.17$ & Present Work \\
\hline
\end{tabular}


seen that the values of $\left\langle N_{b}\right\rangle$ obtained in ${ }^{32} \mathrm{~S}$-Em interactions at $200 \mathrm{AGeV}$ are slightly larger than the values obtained by S. Dhamija et al. and A. Dabrowska et al. This discrepancy may be due to the different criteria of ionization chosen for black tracks by S. Dhamija and A. Dabrowska. The values of $\left\langle N_{g}\right\rangle$ compare reasonably well. It can be observed from the results shown in the table that the value of $\left\langle N_{g}\right\rangle$ depends weakly with increasing mass of the projectiles as well as energy of the projectiles, whereas the values of $\left\langle N_{g}\right\rangle$ do not exhibit any such trends. The increasing trend is missing in the values of $\left\langle N_{b}\right\rangle$ because slow particles are produced due to evaporation of excited residual nucleus. We also study the dependence of the average multiplicities on projectile mass, $A_{p}$, using the following power law:

$$
\left\langle N_{i}\right\rangle=\alpha_{i}\left(A_{p}\right)^{\beta_{j}}
$$

where $i=b, g, h$, and $\left\langle N_{i}\right\rangle$ represents the mean multiplicity. The values of the slope $(\beta)$ for black, grey, and heavy tracks obtained from the least - squares fits are given as $(-0.0054 \pm 0.009),(0.1407 \pm 0.008)$ and $(0.1125$ \pm 0.016 ) respectively. The dependence of $\left\langle N_{b}\right\rangle,\left\langle N_{g}\right\rangle$ and $\left\langle N_{h}\right\rangle$ on the projectile mass number, $A_{p}$, is shown in Figures 5(a)-(c). It has been reported that the values of $\beta_{g}$ for $\left\langle N_{g}\right\rangle$ is slightly higher than the corresponding values in case of hadron-nucleus interactions [18] [19]. The observation may indicate that the ${ }^{32} \mathrm{~S}$ projectile at a given impact parameter are an extended object rather than a point object as in the case of a hadron beam. The variation of $\left\langle N_{b}\right\rangle$ with $A_{p}$ is shown in Figure 5(a), which is almost independent of the projectile mass number and its energy. This constancy of $\left\langle N_{b}\right\rangle$ indicates that the average excitation of the residual target nucleus has reached its saturation at present projectile energy. The strong dependence of the total charged secondary particles on the masses of colliding nucleus are due to the increase in the overlapping region of the two interacting nuclei.

Table 2 presents the mean multiplicities of black, grey and heavily ionizing particles for ${ }^{32}$ S-emulsion inelastic interactions at $200 \mathrm{AGeV}$ for different $N_{h}$-intervals. It is found that mean multiplicities of slow particles increases with increase of centrality of collisions. A regular pattern in the values of the ratio $\left\langle N_{b}\right\rangle /\left\langle N_{g}\right\rangle$ has been recorded from the table except for CNO $\left(2 \leq N_{h} \leq 7\right)$ events which is slightly higher.

The average values of dispersion $D\left(N_{g}\right),\left\langle N_{b}\right\rangle /\left\langle N_{h}\right\rangle$ and $D /\left\langle N_{g}\right\rangle$ for different $N_{h}$-intervals of ${ }^{32} \mathrm{~S}$-Em in-

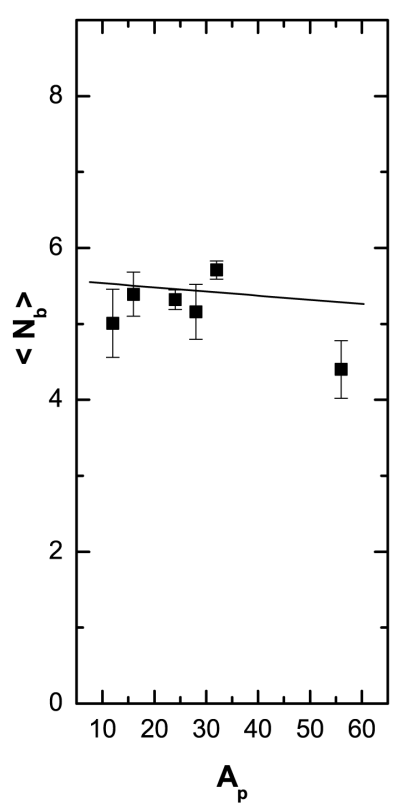

(a)

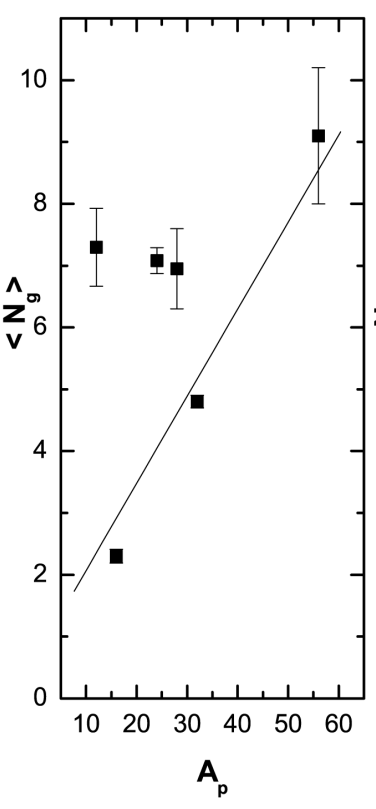

(b)

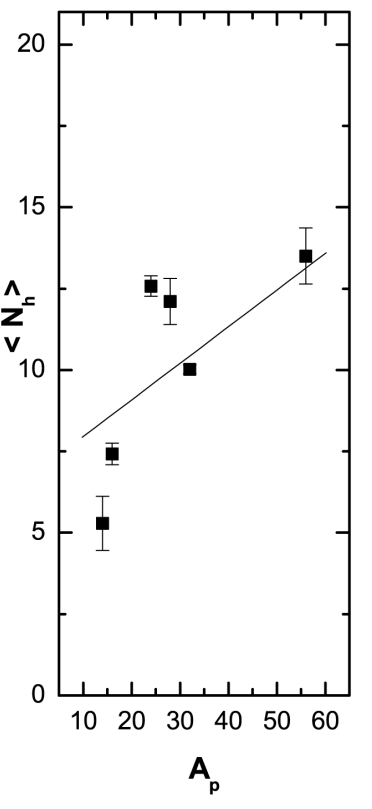

(c)

Figure 5. Variation of $\left\langle N_{b}\right\rangle,\left\langle N_{g}\right\rangle$ and $\left\langle N_{h}\right\rangle$ as a function of projectile mass number $\left(A_{p}\right)$ at different energies. 
Table 2. Average values of $\left\langle N_{g}\right\rangle,\left\langle N_{b}\right\rangle,\left\langle N_{h}\right\rangle$ in ${ }^{32}$ S-Em interactions along with different $N_{h}$ intervals at $200 \mathrm{AGeV}$.

\begin{tabular}{ccccc}
\hline Group of events & $\left\langle N_{g}\right\rangle$ & $\left\langle N_{b}\right\rangle$ & $\left\langle N_{h}\right\rangle$ & $\left\langle N_{b}\right\rangle /\left\langle N_{g}\right\rangle$ \\
\hline All ${ }^{32} \mathrm{~S}-\mathrm{E}_{\mathrm{m}}$ & $3.14 \pm 0.10$ & $6.67 \pm 0.14$ & $9.81 \pm 0.17$ & $2.12 \pm 0.03$ \\
$2 \leq N_{h} \leq 7$ & $1.54 \pm 0.12$ & $4.04 \pm 0.20$ & $5.58 \pm 0.22$ & $2.62 \pm 0.08$ \\
$8 \leq N_{h} \leq 20$ & $3.81 \pm 0.13$ & $7.77 \pm 0.18$ & $11.58 \pm 0.22$ & $2.03 \pm 0.03$ \\
$N_{h}>20$ & $6.50 \pm 0.42$ & $13.87 \pm 0.67$ & $20.37 \pm 0.78$ & $2.13 \pm 0.13$ \\
$N_{h} \geq 8$ & $3.84 \pm 0.13$ & $7.83 \pm 0.17$ & $11.67 \pm 0.22$ & $2.03 \pm 0.043$ \\
\hline
\end{tabular}

teractions at $200 \mathrm{AGeV}$ are given in Table 3. From the table it may be clearly noticed that as the impact parameter decreases (the degree of disintegration of the target nuclei increases), the ratio of the number of slow evaporated particles $\left(N_{b}\right)$ to heavily ionizing particles $\left(N_{h}\right)$ i.e. the $\left\langle N_{b}\right\rangle /\left\langle N_{h}\right\rangle$ is nearly constant. This result contradicts the results obtained by Antonchilk et al. [20] for $7 \leq N_{h} \leq 27$ and $N_{h} \geq 28$ respectively.

\subsection{Multiplicity Correlations}

Multiplicity correlations among the heavily ionizing particles produced in nucleus-nucleus collisions have been widely studied which help to investigate the mechanism of particle production. In order to examine the behaviour of multiplicity correlations of secondary particles produced in nucleus- nucleus collisions, we have studied the correlations in the interactions of ${ }^{32} \mathrm{~S}-\mathrm{Em}$ at $200 \mathrm{AGeV}$. Generally, the experimental results have been analyzed by using linear fits of the type:

$$
\left\langle N_{i}\left(N_{j}\right)\right\rangle=a_{i j} N_{j}+b_{i j}
$$

where $N_{i}, N_{j}=N_{g}, N_{b}$ and $N_{h}$ with $i \neq j$. The values of inclination coefficients, $a_{i j}$ and intercepts, $b_{i j}$ are given in Table 4. The behaviour of multiplicity correlations of secondary particles produced in ${ }^{32} \mathrm{~S}$-Em interactions at $200 \mathrm{AGeV}$ is shown in Figures 6(a)-(c) along with their linear fits using least square method. From these plots following conclusions may be drawn.

i) A clear saturation in the values of $\left\langle N_{b}\right\rangle$ is observed in $\left\langle N_{b}\right\rangle$ vs $N_{g}$ plot for values of $N_{g}$ beyond $\sim 10$. A similar result was obtained by Otterlund [18] for proton-nucleus interactions over a wide range of energy. This means correlation does not depend upon mass of the projectiles and the contribution of the recoiling nucleons towards the excitation energy of the residual nucleus is approximately the same for P- nucleus and nucleus-nucleus interactions.

ii) Variation of $\left\langle N_{g}\right\rangle$ with $N_{b}$ is similar to that of $\left\langle N_{b}\right\rangle$ with $N_{g}$. However, the saturations are not statistically significant. Also $\left\langle N_{g}\right\rangle$ displays a linear dependence on $N_{h}$ in the whole range of $N_{h}$.

iii) The values of $\left\langle N_{h}\right\rangle$ increases with the increase of $N_{b}$ and $N_{g}$ in the whole range of $N_{b}$ and $N_{g}$ in nucleusnucleus collisions.

\subsection{Target Size Dependence of $\left\langle N_{b}\right\rangle,\left\langle N_{g}\right\rangle$ and $\left\langle N_{h}\right\rangle$}

In order to see the dependence of the average multiplicities on the mass numbers of the target nuclei, the following relation has been used as:

$$
\left\langle N_{j}\right\rangle=K A^{\alpha}
$$

where “j” stands for black, grey and heavy particles respectively.

The variation of average multiplicities on masses of the target nuclei is shown in Figure 7. The coefficients $K$ and $\alpha$, are determined from the least-square fit using the experimental data of the present study. The values of these coefficients are given in Table 5. The results depict that the multiplicities of black particles are nearly proportional to linear dimensions of target nuclei. The multiplicities of grey particles are characterized by extremely weak target size dependence. 


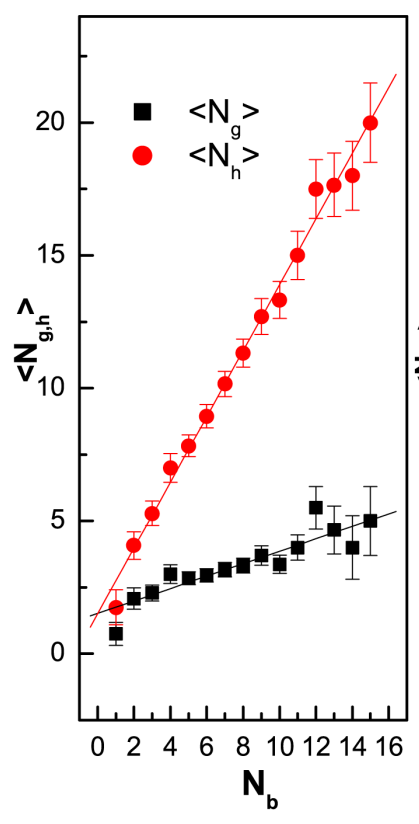

(a)

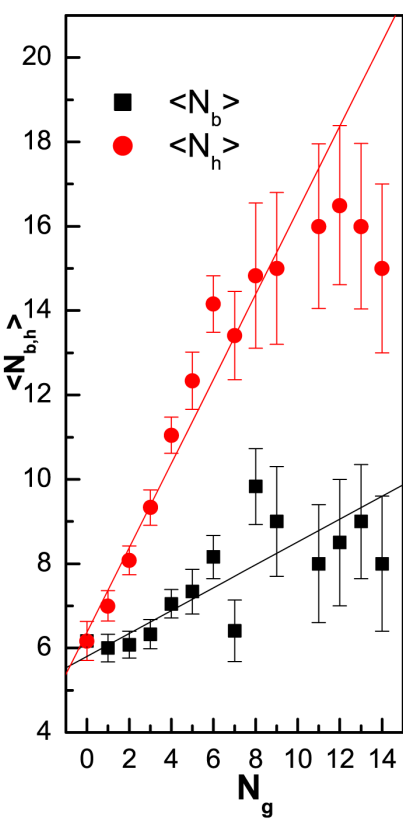

(b)

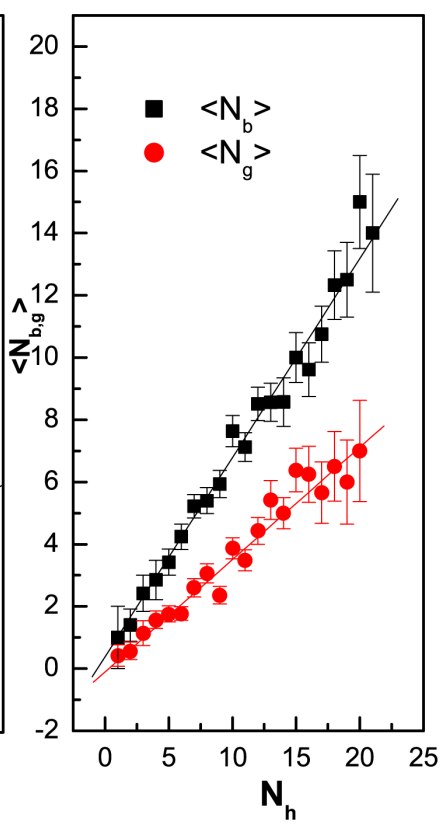

(c)

Figure 6. Multiplicity correlations of various charged particles produced in the interactions of ${ }^{32} \mathrm{~S}-\mathrm{Em}$ at $200 \mathrm{AGeV}$.

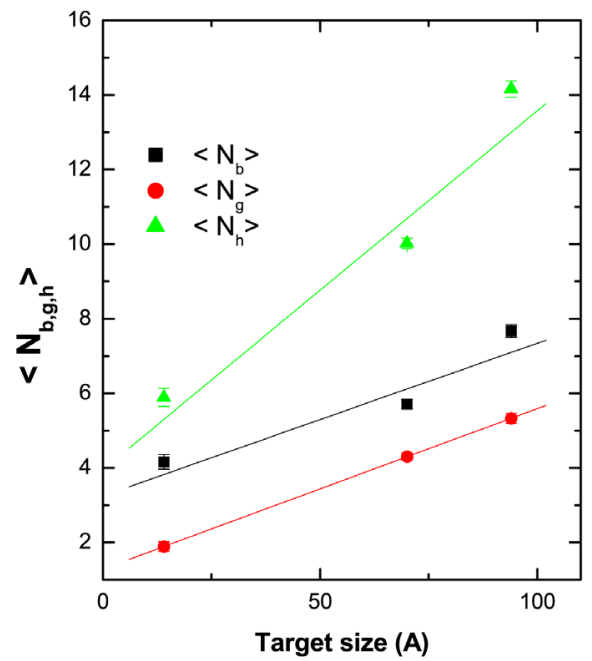

Figure 7. Variation of $\left\langle N_{b}\right\rangle,\left\langle N_{g}\right\rangle$ and $\left\langle N_{h}\right\rangle$ with the size of the target $A$.

Table 3. Values of $D\left(N_{g}\right)$ and $\frac{\left\langle N_{b}\right\rangle}{\left\langle N_{h}\right\rangle}$.

\begin{tabular}{ccccc}
\hline Group of events & $D\left(N_{g}\right)$ & $\frac{\left\langle N_{b}\right\rangle}{\left\langle N_{h}\right\rangle}$ & $\frac{D}{\left\langle N_{g}\right\rangle}$ & $\frac{\left\langle N_{g}\right\rangle}{D}$ \\
\hline${ }^{32}$ S-Em & $2.07 \pm 0.13$ & $0.67 \pm 0.05$ & $0.65 \pm 0.06$ & $1.51 \pm 0.04$ \\
$2 \leq N_{h} \leq 7$ & $1.17 \pm 0.10$ & $0.72 \pm 0.06$ & $0.75 \pm 0.08$ & $1.31 \pm 0.06$ \\
$8 \leq N_{h} \leq 20$ & $1.95 \pm 0.12$ & $0.67 \pm 0.04$ & $0.51 \pm 0.04$ & $1.95 \pm 0.08$ \\
$N_{h}>20$ & $1.65 \pm 0.15$ & $0.68 \pm 0.07$ & $0.25 \pm 0.02$ & $3.93 \pm 0.10$ \\
$N_{h} \geq 8$ & $1.96 \pm 0.11$ & $0.67 \pm 0.06$ & $0.51 \pm 0.04$ & $1.95 \pm 0.08$ \\
\hline
\end{tabular}


Table 4. Values of inclination coefficients $a_{i j}$ and intercepts $b_{i j}$ in multiplicity correlation in ${ }^{32} \mathrm{~S}$-Em interactions at 200 $\mathrm{AGeV}$.

\begin{tabular}{ccccccc}
\hline$N_{j}$ & \multicolumn{7}{c}{$\boldsymbol{a}_{\boldsymbol{i j}}$} & & \multicolumn{1}{c}{$\boldsymbol{b}_{\boldsymbol{i j}}$} & \\
\cline { 2 - 6 } & $\left\langle N_{b}\right\rangle$ & $\left\langle N_{g}\right\rangle$ & $\left\langle N_{h}\right\rangle$ & $\left\langle N_{b}\right\rangle$ & $\left\langle N_{g}\right\rangle$ & $\left\langle N_{h}\right\rangle$ \\
\hline$N_{b}$ & $\ldots$ & $0.23 \pm 0.03$ & $1.23 \pm 0.03$ & $\ldots$ & $1.51 \pm 0.22$ & $1.50 \pm 0.20$ \\
$N_{g}$ & $0.27 \pm 0.07$ & $\ldots$ & $1.00 \pm 0.09$ & $5.79 \pm 0.34$ & $\ldots$ & $6.37 \pm 0.26$ \\
$N_{h}$ & $0.64 \pm 0.02$ & $0.36 \pm 0.02$ & $\ldots$ & $0.38 \pm 0.01$ & $-0.10 \pm 0.06$ & $\ldots$ \\
\hline
\end{tabular}

Table 5. Values of coefficients $K$ and $\alpha$.

\begin{tabular}{ccc}
\hline$N_{j}$ & $K$ & $\alpha$ \\
\hline$\left.N_{b}\right\rangle$ & $3.95 \pm 0.27$ & $0.09 \pm 0.003$ \\
$\left\langle N_{g}\right\rangle$ & $3.24 \pm 0.22$ & $0.04 \pm 0.003$ \\
$\left\langle N_{h}\right\rangle$ & $1.28 \pm 0.15$ & $0.04 \pm 0.002$ \\
\hline
\end{tabular}

\subsection{Angular Distribution of Slow Particles}

Figure 8(a) and Figure 8(b) shows the normalized angular distributions for black and grey particles emitted in ${ }^{32} \mathrm{~S}-\mathrm{Em}$ collisions at $200 \mathrm{AGeV}$ along with the results obtained by other workers [18] [21] for different projectiles and energies. It has been observed from the figure that the distributions are independent of projectile mass as well as the energy of the projectile. The production mechanism of the black particles, based on nuclear evaporation processes, are isotropically distributed in the rest frame of target nucleus and the distribution is influenced by a strong electromagnetic field of relativistic projectiles, which is almost symmetric about $\theta \approx 90^{\circ}$ in the laboratory system. Whereas the production mechanism of grey particles is based on inter-nuclear cascade processes and these particles are mainly emitted in forward direction. The forward and backward hemispheres are defined as the regions where emission angles are less than $90^{\circ}\left(\theta<90^{\circ}\right)$ and greater than $90^{\circ}\left(\theta>90^{\circ}\right)$ respectively. The forward (F) to backward (B) ratio for these distributions are calculated and presented in Table 6. It is clearly seen from the table that the F/B ratio shows the same behavior and its value for grey particles is more than black particles. Also the F/B ratio shows a weak dependence on the mass of projectile. The differences in the angular distributions of black and grey particles point to the fact that these particles originate in two different processes, the subsequent evaporation and the initial interaction of target nucleus.

\subsection{KNO Scaling}

Recently [24]-[26] the investigation of nuclear fragments produced in high energy nucleus-nucleus collisions shows that the multiplicity distributions of target fragments; black and grey particles can be described by KobaNielsen-Olesen scaling [27]. Koba, Nielsen and Olesen have predicted that the multiplicity distributions of the produced particles in high-energy hadron-hadron collisions should obey a simple scaling law known as KNO scaling when expressed in terms of the scaling variable $Z(=N /\langle N\rangle)$. If $P_{n}(\mathrm{~s})$ represents the probability for the production of $n$ charged particles in an inelastic hadron-hadron collision at a centre of mass energy $\sqrt{ } s$, then the multiplicity distributions in high energy collision obey a scaling law:

$$
P_{n}(s)=\frac{\sigma_{n}(s)}{\sigma_{\text {inel }}(s)}=\frac{1}{\langle N\rangle} \Psi\left(\frac{N}{\langle N\rangle}\right)=\frac{1}{\langle N\rangle} \Psi(Z)
$$

where $\sigma_{n}(s)$ is the partial cross-section for the production of $\mathrm{n}$ charged particles, $\sigma_{\text {inel }}$ is the total inelastic cross-section and $\langle N\rangle$ is the average number of charged particles produced. The KNO scaling thus implies that the multiplicity distribution is universal and $\psi(z)$ is an energy independent function at sufficiently high energies when expressed in terms of scaling variable $Z$. 


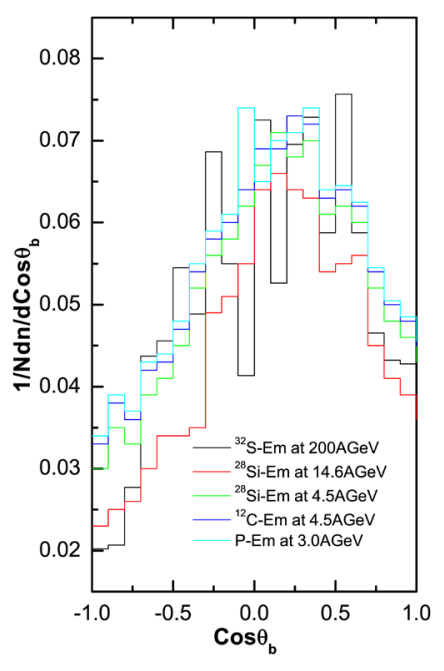

(a)

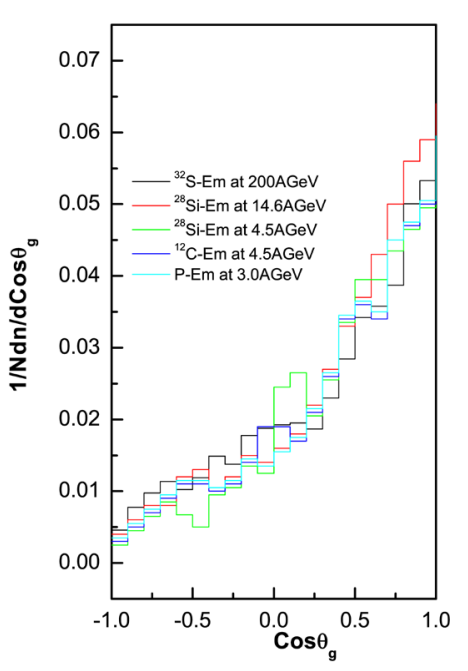

(b)

Figure 8. The normalized angular distributions of (a) black and (b) grey particles in ${ }^{32} \mathrm{~S}$-Em interactions at $200 \mathrm{AGeV}$ along with other results.

Table 6. The values of F/B ratio for the angular distribution of produced particles in nuclear collisions.

\begin{tabular}{cccccc}
\hline Beam & Momentum AGeV/c & Grey & Black & Sample & Ref. \\
\hline \multirow{2}{*}{ Sulphur } & 200 & $3.25 \pm 0.12$ & $1.25 \pm 0.10$ & ${ }^{32} \mathrm{~S}$-Em & Present Work \\
& & $3.05 \pm 0.12$ & $1.32 \pm 0.11$ & $2 \leq N_{h} \leq 7$ & Present Work \\
Silicon & 14.6 & $3.85 \pm 0.14$ & $1.46 \pm 0.09$ & $N_{h} \geq 8$ & Present Work \\
Silicon & 4.5 & $4.78 \pm 0.21$ & $1.37 \pm 0.09$ & ${ }^{28} \mathrm{Si-Em}$ & {$[24]$} \\
Carbon & 4.5 & $5.29 \pm 0.24$ & $1.84 \pm 0.06$ & ${ }^{28} \mathrm{Si-Em}$ & {$[21]$} \\
Alpha & 4.5 & $4.69 \pm 0.18$ & $1.49 \pm 0.04$ & ${ }^{12} \mathrm{C}$-Em & {$[21]$} \\
Proton & 3.0 & $3.00 \pm 0.10$ & $1.42 \pm 0.04$ & ${ }^{4} \alpha$-Em & {$[22]$} \\
\hline
\end{tabular}

It has been found by various workers that the empirical expression for $\psi(z)$ in hadron-hadron and hadronnucleus interactions obeys the semi-inclusive KNO scaling starting from few GeV. It is desirable to make similar studies in nucleus-nucleus collisions as it is expected that nucleus-nucleus collisions (A-A) at these energies can be visualized as superposition of nucleon-nucleon collisions. Several workers [24] [28]-[30] have reported that the validity of KNO scaling holds for the projectile helium particle and black tracks in heavy-ion interactions at Dubna, Bevatron, CERN and AGS energies. It has been shown [26] [31] that the multiplicity distributions of produced black and grey fragments obtained from the events of different projectiles over a wide range of energies in nucleus-nucleus collisions can be described by a KNO scaling law. These distributions can be represented by a universal function of the following form:

$$
\Psi(Z)=A Z \exp (-B Z)
$$

where $A$ and $B$ are constants.

In the present work an attempt has been made to study the KNO scaling for the multiplicity distribution of slow and fast target associated particles produced in ${ }^{32}$ S-emulsion collisions at $200 \mathrm{AGeV}$. A plot of $\psi(z)$ as a function of the scaling variable $Z(=N /\langle N\rangle)$ for these medium energy target associated protons is shown in Figure 9(a) and Figure 9(b). The experimental points for ${ }^{12} \mathrm{C}$ and ${ }^{28} \mathrm{Si}$ at $4.5 \mathrm{AGeV}[8],{ }^{28} \mathrm{Si}$ at $14.6 \mathrm{~A} \mathrm{GeV}$ [8] and ${ }^{16} \mathrm{O}$ at $3.7 \mathrm{AGeV}$ and $60 \mathrm{AGeV}$ [9] respectively are also shown in the same figure. The solid curve in the figure is well represented by Equation (6). 


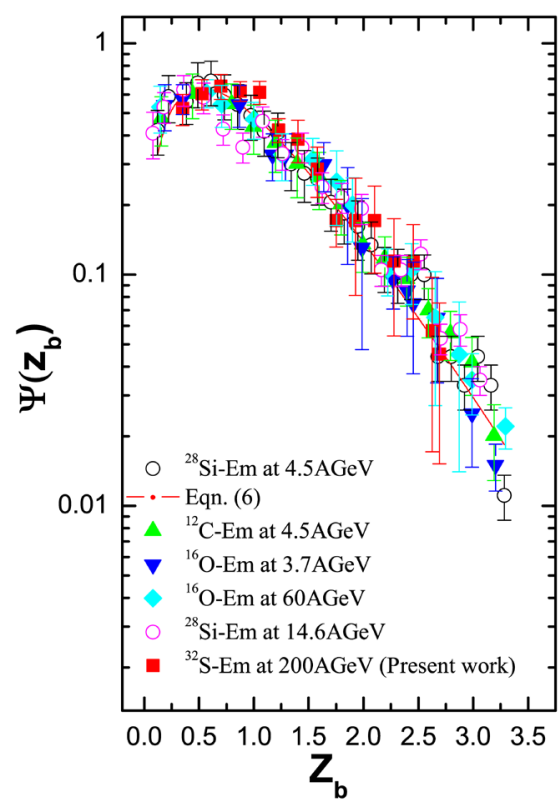

(a)

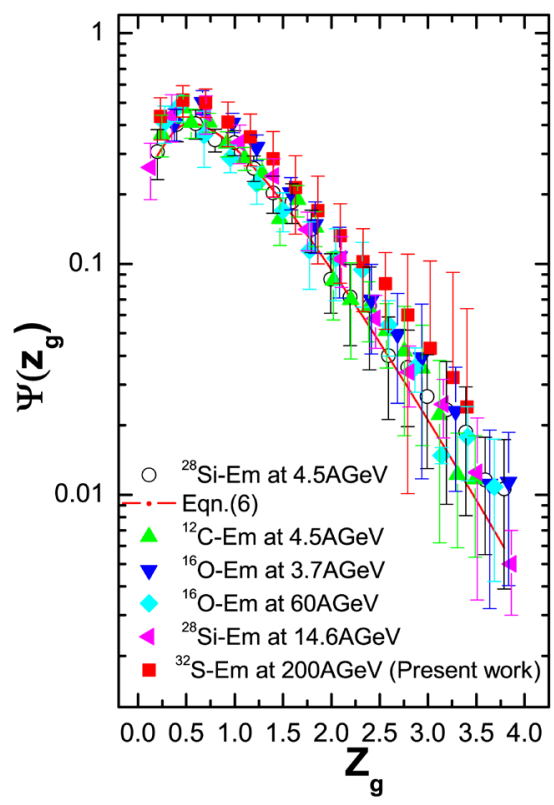

(b)

Figure 9. Multiplicity distribution of (a) slow target associated protons and (b) fast target associated protons in terms of KNO scaling in the interactions of ${ }^{28} \mathrm{Si}-\mathrm{Em}$ at 4.5 and $14.6 \mathrm{AGeV},{ }^{12} \mathrm{C}-\mathrm{Em}$ at $4.5 \mathrm{AGeV}$ and ${ }^{16} \mathrm{O}-\mathrm{Em}$ at 3.7 and $60 \mathrm{AGeV}$ with the present work of ${ }^{32} \mathrm{~S}-\mathrm{Em}$ at $200 \mathrm{AGeV}$.

It is easily noticed from the figures that the multiplicity distributions of slow and fast target associated protons in nucleus-nucleus collisions at different energies are well described by Equation (6) for the different projectiles and seem to satisfy the scaling function. The best values of $A$ and $B$ used in Equation (6) are found to be $6.21 \pm$ $0.85,2.65 \pm 0.10$ and $5.26 \pm 0.90,2.89 \pm 0.13$ respectively for slow and fast target associated protons. The values of corresponding $\chi^{2} / \mathrm{DOF}$ are found to be 0.63 and $0.79 \pm 0.04$ respectively for slow and fast target associated protons which indicates that the fitting is good for different projectiles at different energies in case of slow protons but a small deviation from exact scaling can be seen for fast target associated protons in Figure 9(b). It is difficult to give any physical explanation of the multiplicity scaling for slow and fast protons and hence can be regarded as an empirical observation.

\subsection{Negative Binomial Distribution of Black, Grey and Heavy Particles}

The studies of multiplicity distribution in high energy heavy ion collisions have revealed some striking phenomenon which can be described by various models [27] [32]-[34]. Among them, the most spectacular and well known is the negative binomial distribution (NBD) model [34]. The NB law has been successful in describing the multiplicity results in recent high energy experiments. In Figures 10(a)-(c) the multiplicity distributions of black, grey and heavy particles in the interactions of ${ }^{32} \mathrm{~S}$-Em at $200 \mathrm{AGeV}$ are shown with the corresponding best NB fits. The values of the free parameters of negative binomial distributions " $k$ " and " $n$ " and the $\chi^{2} / \mathrm{DOF}$ obtained using the CERN MINUIT curve fitting program are given in Table 7. From the table it can be concluded that the multiplicity distributions of secondary particles produced in ${ }^{32} \mathrm{~S}$-Em at $200 \mathrm{AGeV}$ interactions can be fitted quite satisfactorily by the NBD having the form

$$
P(n,\langle n\rangle, k)=k(k+1) \cdots(k+n-1)\left(\frac{\langle n\rangle / k}{1+\langle n\rangle / k}\right)^{n}\left(\frac{1+\langle n\rangle / k}{n !}\right)^{k}
$$

where $n$ and $\langle n\rangle$ represent respectively the multiplicity and mean multiplicity, the value of $\mathrm{k}$ is determined from:

$$
\frac{1}{k}+\frac{1}{n}=\left(\frac{D(n)}{\langle n\rangle}\right)^{2}
$$




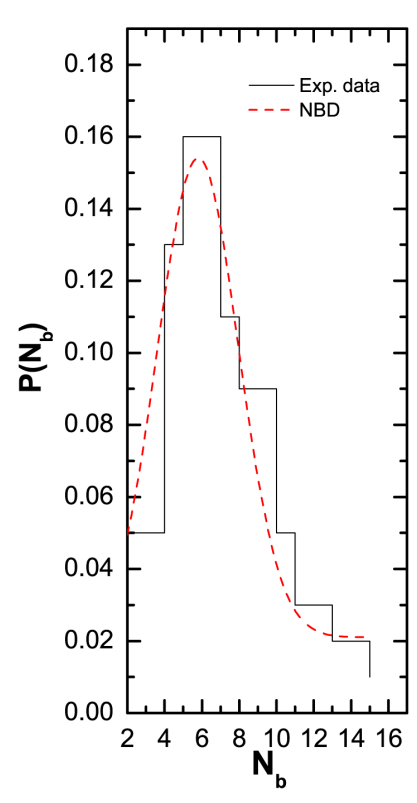

(a)

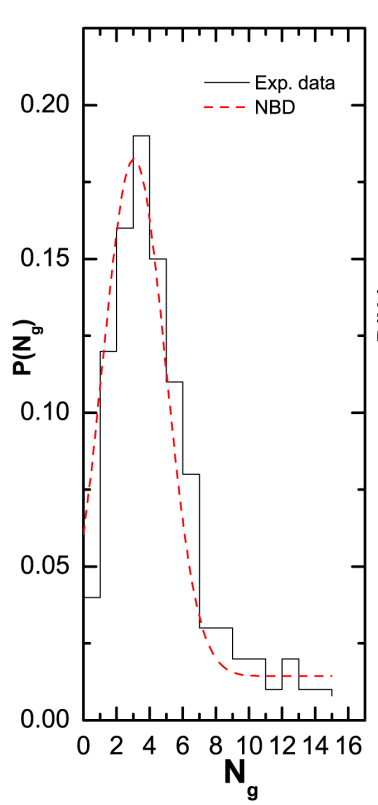

(b)

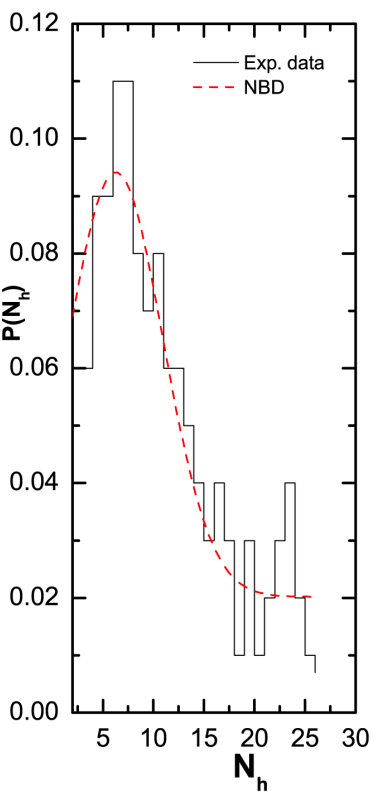

(c)

Figure 10. The Multiplicity distributions of (a) black particles (b) grey particles and (c) heavily ionizing particles with NB fits in ${ }^{32} \mathrm{~S}$-Em interactions at $200 \mathrm{AGeV}$.

Table 7. Values of free parameters of NBD.

\begin{tabular}{cccc}
\hline$N_{i}$ & $n$ & $k$ & $\chi^{2} / \mathrm{DOF}$ \\
\hline$N_{b}$ & $4.32426 \pm 0.53367$ & $0.722 \pm 0.10868$ & 0.32 \\
$N_{g}$ & $3.79438 \pm 0.22139$ & $0.80073 \pm 0.05018$ & 0.30 \\
$N_{h}$ & $9.3698 \pm 1.29225$ & $0.86879 \pm 0.12831$ & 0.48 \\
\hline
\end{tabular}

\section{Conclusions}

The following conclusions may be drawn from the present study.

i) It is observed that the peaks $N_{b}, N_{g}$, and $N_{h}$ of distributions appear in the lower values of $N_{b}, N_{g}$, and $N_{h}$ and all the distributions are essentially independent of incident energy and projectile masses. It is also clear that the target associated particles have a weak dependence on the projectile mass number $A_{p}$. Further, the multiplicity distributions of slow and fast protons produced in nucleus-nucleus interactions give a clear indication that no significant differences are observed regarding the mechanism of their production with energy.

ii) It is found that mean multiplicity of slow particles increases with increase of centrality of collisions. A regular pattern in the values of the ratio $\left\langle N_{b}\right\rangle /\left\langle N_{g}\right\rangle$ has been observed except for CNO $\left(2 \leq N_{h} \leq 7\right)$ events.

iii) It can be observed that the value of $\left\langle N_{g}\right\rangle$ depends weakly with increasing mass of the projectiles as well as energy of the projectiles. The values of $\left\langle N_{b}\right\rangle$ do not exhibit any such trends.

iv) It may be noticed that as the impact parameter decreases (the degree of disintegration of the target nuclei increases), the ratio of the number of slow evaporated particles $\left(N_{b}\right)$ to heavily ionizing particles $\left(N_{h}\right)$ i.e. the $\left\langle N_{b}\right\rangle /\left\langle N_{h}\right\rangle$ is approximately constant.

v) The multiplicity correlations of secondary particles produced in nucleus-nucleus collisions are similar to hadron-nucleus collisions and could be represented by a linear dependence.

vi) It has been found that multiplicities of black particles are nearly proportional to linear dimensions of target nuclei whereas the multiplicities of grey particles are characterized by extremely weak target size dependence.

vii) The angular distributions of the black particles, based on nuclear evaporation processes, are isotropically 
distributed in the rest frame of target nucleus and the distribution is influenced by a strong electromagnetic field of relativistic projectiles, which is almost symmetric about $\theta \approx 90^{\circ}$ in the laboratory system. The angular distribution of grey particles and their production are based on inter-nuclear cascade processes. These fast protons are mainly emitted in forward direction.

viii) The multiplicity distributions of slow and fast target associated protons produced in ${ }^{32} \mathrm{~S}$-Em collisions at $200 \mathrm{AGeV}$ along with other experimental data at various energy ranges exhibit a KNO scaling within experimental errors and a small scaling violations are found.

ix) Also the multiplicity distributions of secondary particles produced in ${ }^{32} \mathrm{~S}-\mathrm{Em}$ at $200 \mathrm{AGeV}$ interactions can be fitted quite satisfactorily by the NBD.

\section{Acknowledgements}

We would like to express our thanks to Professor P.L. Jain of SUNY at Buffalo, USA for providing the exposed and developed emulsion plates for the present analysis.

\section{References}

[1] EMU01 Collaboration, Adamovich, M.I., Aggarwal, M.M., Alexandrov, Y.A., Andreeva, N.P., Anson, Z.V., Arora, R., et al. (1991) Physics Letters B, 262, 369-374. http://dx.doi.org/10.1016/0370-2693(91)91580-O

[2] Dhamija, S., Aggarwal, M.M. and Bhatia, V.S. (2001) Modern Physics Letters A, 16, 1801. http://dx.doi.org/10.1142/S021773230100408X

[3] Abd-Allah, N.N. and Mohery, N. (2001) Turkish Journal of Physics, 25, 109.

[4] Rasool, M.H., Ahmad, S. and Ayaz, M. (2015) Journal of Korean Physical Society, 67, 448-457. http://dx.doi.org/10.3938/jkps.67.448

[5] Rasool, M.H., Ayaz Ahmad, M. and Ahmad, S. (2015) Chaos, Solitons and Fractals, 81, 197-202. http://dx.doi.org/10.1016/j.chaos.2015.08.027

[6] Rasool, M.H., Ayaz Ahmad, M., Singh, O.V. and Ahmad, S. (2015) Journal of Modern Physics, 6, 1498-1509. http://dx.doi.org/10.4236/jmp.2015.611154

[7] Rasool, M.H., Ayaz Ahmad, M., Bhat, M.A. and Ahmad, S. (2015) Journal of Nuclear Science and Technology, 5, 208-220. http://dx.doi.org/10.4236/wjnst.2015.53021

[8] Ahmad, M.A., Ahmad, S. and Rasool, M.H. (2012) International Journal of Theoretical and Applied Physics, 2, 199-220.

[9] Jain, P.L., Sengupta, K. and Singh, G. (1991) Physical Review C, 44, 844. http://dx.doi.org/10.1103/PhysRevC.44.844

[10] El-Nadi, M., Abdelsalam, A., Hussein, A., Shaat, E., Ali-Mousa, N., Abou-Mousa, Z. and El-Falaky, E. (1995) Il Nuovo Cimento A, 108, 831-842. http://dx.doi.org/10.1007/BF02731024

[11] DGKLMTW Collaboration (1974) JINR Dubna Communication, PL-8313.

[12] Antonchik, V.A., et al. (1980) Soviet Journal of Nuclear Physics, 32, 164-172.

[13] Chernov, G.M., Gulamov, K.G., Gulyamov, U.G., Nasyrov, S.Z. and Svechnikova, L.N. (1977) Nuclear Physics A, 280, 478-490. http://dx.doi.org/10.1016/0375-9474(77)90616-9

[14] Ahmad, S., Ayaz Ahmad, M., Tariq, M. and Zafar, M. (2009) International Journal of Modern Physics E, 18, 1929-1944. http://dx.doi.org/10.1142/S0218301309013968

[15] Dabrowska, A., Hołyński, R., Jurak, A., Olszewski, A., Szarska, M., Trzupek, A., et al. (1993) Physical Review D, 47, 1751-1761.

[16] Liu, F.H. (2002) Chinese Journal of Physics, 40, 159-167.

[17] El-Nadi, M., Uzhinskii, V.V., Sherif, M.M., Abd-Elsalam, A., El-Nagdy, M.S., Yasin, M.N., et al. (2004) International Journal of Modern Physics E, 13, 619-630. http://dx.doi.org/10.1142/S0218301304002363

[18] Otterlund, I., Stenlund, E., Andersson, B., Nilsson, G., Adamovic, O., Juric, M., et al. (1978) Nuclear Physics B, 142, 445-462. http://dx.doi.org/10.1016/0550-3213(78)90223-7

[19] Hegab, M.K. and Hufner, J. (1982) Nuclear Physics A, 384, 353-370. http://dx.doi.org/10.1016/0375-9474(82)90340-2

[20] Antonchilk, V.A., et al. (1984) Soviet Journal of Nuclear Physics, 39, 774.

[21] Tariq, M., Ahmad, S., Tufail, A. and Zafar, M. (1994) Il Nuovo Cimento A, 107, 2687-2699.

http://dx.doi.org/10.1007/BF02730949

[22] DGX \& MTW Coll. (1974) JINR Dubna Communication. Pl-8313. 
[23] Bogdanski, M., et al. (1969) Helvetica Physica Acta, 13, 485.

[24] Ayaz Ahamd, M. and Ahmad, S. (2012) Ukrainian Journal of Physics, 57, 1205-1213.

[25] Liu, F.H. and Panebratsev, Y.A. (1998) Il Nuovo Cimento A, 111, 1219-1224.

[26] Liu, F.H. (2000) Physical Review C, 62, Article ID: 024613. http://dx.doi.org/10.1103/PhysRevC.62.024613

[27] Koba, Z., Nielsen, H.B. and Olesen, P. (1972) Nuclear Physics B, 40, 317-334. http://dx.doi.org/10.1016/0550-3213(72)90551-2

[28] El-Nadi, M., El-Nagdy, M.S., Ali-Mossa, N., Abdelsalam, A., Abdalla, A.M. and Abdel-Halim, S.M. (2002) Journal of Physics G: Nuclear and Particle Physics, 28, 1251-1258. http://dx.doi.org/10.1088/0954-3899/28/6/308

[29] Liu, F.H. (2003) Chinese Journal of Physics, 41, 486-496.

[30] Singh, G., Sengupta, K. and Jain, P.L. (1990) Physical Review C, 42, 1757-1759. http://dx.doi.org/10.1103/PhysRevC.42.1757

[31] Sherif, M.M., Hegab, M.K., Abdelsalam, A., El-Sharkawy, S.A. and Tawfik, A.M. (1993) International Journal of Modern Physics E, 2, 835-843. http://dx.doi.org/10.1142/S0218301393000388

[32] Ghosh, D., Ghosh, A., Mukhopadhyay, A. and Roy, J. (1989) Nuclear Physics A, 491, 684-693. http://dx.doi.org/10.1016/0375-9474(89)90525-3

[33] Giovannmini, A. and Van Hove, L. (1986) Zeitschrift für Physik C: Particles and Fields, 30, 391-400. http://dx.doi.org/10.1007/BF01557602

[34] Chew, C.K., Kiang, D. and Zhou, H. (1987) Physics Letters B, 186, 411-415. http://dx.doi.org/10.1016/0370-2693(87)90318-2 